\section{NIV-Basiskurs der ERS 2017}

Im Jahr 2007 hat die European Respiratory Society (ERS) erstmals den Postgraduiertenkurs „Non Invasive Ventilation“ (NIV) unter der gemeinsamen Leitung von Prof. Dr. Tobias Welte und Prof. Dr. Bernd Schönhofer in Hannover ausgerichtet. Dieser Kurs war der Beginn einer erfolgreichen Tradition.

In den folgenden 10 Jahren fand der NIVKurs noch 4-mal (2009, 2011, 2014 und 2017) in Hannover mit dem Themenschwerpunkt „NIV bei akuter und chronischer ventilatorischer Insuffizienz“ statt. Alle Kurse waren mit ca. 80 Teilnehmern ausgebucht. Die Attraktivität des Kurses liegt in der sehr guten Vermittlung des rasch wachsenden Wissens zur akuten Applikation der NIV und in der chronischen Anwendung in Form der außerklinischen Beatmung. Im Jahr 2014 wurde von der ERS- School entschieden, das komplexe Themenfeld zukünftig in 2 Kurse zu splitten: Ein Kurs „Basiswissen (Basics) zur NIV“ in Hannover und ein Kurs für Fortgeschrittene in Mailand. Beim Kurs in Hannover vom 2. bis 3. Februar 2017 kamen die Teilnehmer aus 22 Ländern.

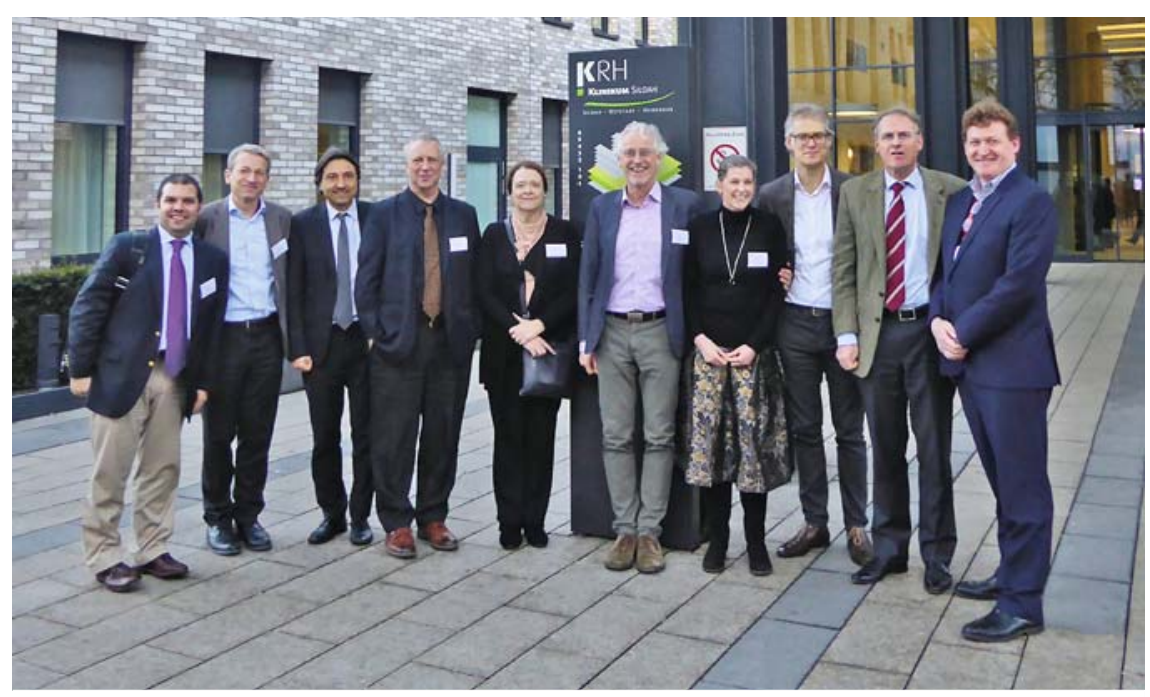

Dr. Miguel Gonçalves (Porto), PD Dr. Thomas Köhnlein (Leipzig), Prof. Raffaele Scala (Arezzo), Prof. Jean-Paul Janssens (Genf), Prof. Anita Simonds (London), Prof. Dr. Bernd Schönhofer (Hannover), Dr. Michelle Chatwin (London), Prof. Wolfram Windisch (Köln), Prof. Peter Wijkstra (Groningen) und Prof. Nicholas Hart (London) (v.l.n.r.). Bildquelle: Schönhofer
Der NIV-Kurs in Hannover bietet den Teilnehmern ohne bisher größere Erfahrung mit der Methode einen Überblick über die aktuelle Evidenzlage, klinisch orientiertes Wissen und die technischen Voraussetzungen, um NIV sicher und effektiv im klinischen Alltag anwenden zu können.

Vor diesem Hintergrund gehört es zum Selbstverständnis der ERS-School, dass durch den NIV-Kurs fundierte Kenntnisse zur Anwendung von NIV im akuten und chronischen Setting sowohl den ERS-Mitgliedern als auch Nichtmitgliedern vermittelt werden.

\section{Kursinhalte}

Im Rahmen des zweitägigen ERS-Basiskurses wurde der Lernstoff in unterschiedlichen Formaten, d.h. Vorträgen, interaktiven Diskussionen, Workshops und Hands-on-Kursen präsentiert.

Im Speziellen erlernen die Teilnehmer grundlegende Kenntnisse zur zugrundeliegenden Pathophysiologie und der Anwendung von NIV bei unterschiedlichen akuten und chronischen Grunderkrankungen, die zur ventilatorischen Insuffizienz führen. Vor allem geht es um die differenzierten Geräteeinstellungen, die sich abhängig von restriktiven Erkrankungen der Lunge und des Thorax bzw. obstruktiven Atemwegs- und Lungenerkrankungen deutlich unterscheiden.

Ein spezielles Modul des Kurses behandelt spezifische Aspekte der Gerätetechnik und des Maskenmaterials.

Ein weiteres Themenfeld beinhaltet Ursachen für die ineffektive Anwendung von NIV und deren Problemlösung, wie z.B. Handhabung größerer Luftlecks und Erkennen ineffektiver Beatmung mit adäquatem Monitoring der Ventilation.

Neben der Vermittlung wichtiger Kenntnisse zum Sekretmanagement mit der Cough-assist-Technik wurde das Entlassungsmanagement der Patienten mit NIV ausführlich thematisiert. 


\section{Herausforderungen und Möglichkeiten}

Eine der größten Herausforderungen des NIV-Basiskurses besteht darin, das grundlegende Wissen zu NIV den Teilnehmern aus z.T. völlig unterschiedlichen sozio-ökonomischen Verhältnissen und verschiedenen Erwartungen zu vermitteln. Trotz Zugehörigkeit zu verschiedenen Gesundheitssystemen war die Motivationslage aller Teilnehmer sehr hoch, praktische Fertigkeiten in der Applikation von NIV zu erwerben.

Alle Teilnehmer nahmen die Gelegenheit wahr, im Workshop eigene praktische Erfahrungen an Patienten mit NIV aus der Klinik Pneumologie und Intensivmedizin des Klinikum Siloah zu machen.

Traditionell zeichnet sich der NIV-Kurs in Hannover durch eine angenehm offene und freundliche Atmosphäre zwischen den Experten und den Teilnehmern aus, die besonders im intensiven Informationsaustausch im Frage-AntwortFormat zum Ausdruck kam. Im „come together" am Abend des 1. Kurstages machten die Kursteilnehmer und Fakultätsmitglieder in angenehmer Atmosphäre reichlich Gebrauch davon, internationale Kontakte zu knüpfen und sich auszutauschen.

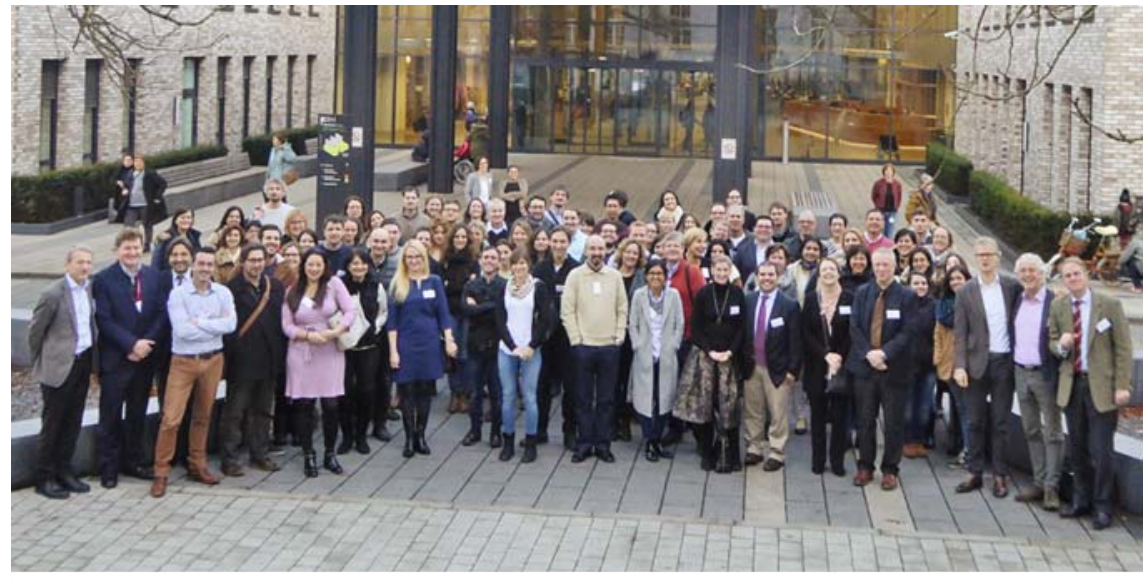

Alle Kursteilnehmer und die Fakultätsmitglieder. Bildquelle: Schönhofer

Am Ende des NIV-Kurses waren die Teilnehmer in der Lage, sicher durch den Dschungel der unterschiedlichen Nomenklatur der Beatmungstechnik zu navigieren und die Konzepte zur NIV beim akuten und chronischen Atmungsversagens unter Berücksichtigung der unterschiedlichen internationalen Perspektiven zu verstehen.

\section{Zukunftspläne}

Die Organisatoren des Kurses und die Fakultätsmitglieder arbeiten seit Jahren eng mit den Verantwortlichen der ERSSchool zusammen. Es ist das erklärte
Ziel aller Beteiligten die Inhalte des NIVKurses in Hannover unter praktischen Aspekten, vor allem im Bereich der Workshops, weiter zu entwickeln. Bereits in den kommenden Monaten wird Prof. Dr. Schönhofer den Antrag auf Ausrichtung des nächsten NIV-Kurses in Hannover im Jahr 2019 an die ERS-School stellen.

Prof. Bernd Schönhofer, Hannover 